\title{
Inverse Filtering of Optical Images
}

\author{
Tamás Dabóczi and Tamás B. Bakó \\ Department of Measurement and Information Systems \\ Technical University of Budapest, H-1521 Budapest, Hungary \\ Phone: +36 1 463-2065, Fax: +36 1 463-4112, email: \{daboczi,bako\}@ mit.bme.hu
}

\begin{abstract}
The quality of images are limited by the performance of the optical system used. The imperfection of the optical system cause distortion of the image. If the distortion is known it can be (partly) compensated. This procedure is called inverse filtering. The problem is, however, ill-posed, which means that the measurement noise is amplified by the inverse filtering process. Suppression of the noise cause bias of the reconstruction. A tradeoff has to be found between the noisy and biased estimates. In this paper the reconstruction of images will be investigated assuming that the distortion of the optical system is known. An algorithm will be introduced to estimate the optimal level of noise suppression of the two dimensional inverse filter.
\end{abstract}

\section{Introduction}

Images are recorded by optical systems. The performance of these systems are limited.

There are different types of errors of such a system. Certain errors exist even by optical systems consisting of perfect spherical lenses. Spherical aberration, coma, chromatic aberration, pincushion distortion etc. [1] belong to this kind of distortion. Other errors are caused by the imperfect manufacturing, or the normal wear of the optical system.

Modern lens systems are compensated for many types of errors. However, they cannot be compensated for all of them. In many cases a certain error can be compensated only for a limited range of usage (e.g.spherical aberration can be compensated for certain object and image distances). The imperfection of manufacturing remains always a limitation. A good example is e.g. the zoom lens of a photo or video camera. The focal length is changed by moving different lenses on different ways together. A small hysteresis of the moving mechanism of the lenses cause blurred (out of focus) images.

Even the user of the optical system can cause distortion by adjusting the system imperfectly. Let think e.g. on pho- tography where the photographer has to adjust the object distance of the lens. If the object distance is set automatically by an "autofocus" circuitry the system can also set an unimportant object to sharp. A typical situation is that two people standing next to each other are out of focus while the tree far behind them is sharp.

\subsection{Modeling the distortion}

An ideal imaging system establishes a point-by-point correspondence between the object and the image. This means that the image of a point is a point in the image plane. In real optical systems, however, the intensity of the light is dispersed over an extended area [2]. The distribution of the intensity is called "point spread function". This effect causes a blurred image. If the spread of the light is space-shift-invariant, and linear, this distortion can be described by a two dimensional convolution, i.e. filtering with the point spread function. A reasonable assumption for the measurement noise is that it is additive at the output of the system:

$$
i(x, y)=o(x, y) * p s f(x, y)+n(x, y),
$$

where $o(x, y)$ denotes the intensity of the object, $i(x, y)$ denotes the intensity of the image, psf $(x, y)$ denotes the space invariant point spread function, $n(x, y)$ denotes the noise and $*$ stands for convolution. The above relationship can be described in the Fourier domain too:

$$
I\left(f_{x}, f_{y}\right)=O\left(f_{x}, f_{y}\right) \operatorname{PSF}\left(f_{x}, f_{y}\right)+N\left(f_{x}, f_{y}\right),
$$

where capital letters stand for the Fourier transform of the corresponding signals. Convolution in space domain becomes multiplication in the Fourier domain.

\subsection{Reconstruction}

The measurement can be (partly) compensated for the distortion, assuming that the point spread function is known and the optical system is linear and space-shift invariant. This procedure is called inverse filtering or deconvolution. 
Inverse filtering is usually an ill-posed problem [3, 4], i.e. measurement noise is amplified in a great extent. The amplified noise has to be suppressed on the price of bias in the estimate. A tradeoff has to be found between the biased and noisy reconstruction. The inverse filter has to compensate the effect of the measurement system in the pass- and attenuation bands, but in the stop band it has to suppress the noise.

$$
O_{e s t}\left(f_{x}, f_{y}\right)=I\left(f_{x}, f_{y}\right) K\left(f_{x}, f_{y}\right),
$$

where $O_{\text {est }}\left(f_{x}, f_{y}\right)$ is the Fourier transform of the estimated intensity of the object and $K\left(f_{x}, f_{y}\right)$ is the transfer function of the inverse filter. The noise reduction is usually controlled by only one parameter (e.g. cutoff frequency of a lowpass filter, regularization parameter, number of maximal iteration etc.).

In order to assure repeatability and omit the subjectivity the level of noise reduction has to be set automatically. In this paper we will describe a model based algorithm which sets automatically the optimal level of noise reduction for two dimensional images. The method is based on a previous work [6], developed for one dimensional time domain transient measurements [7]. The algorithm minimizes the approximate error of the estimate in least squares sense. The technique utilizes rough Fourier domain models of the signals. The models are built automatically from the measurement, and no human interaction is required.

In Section 2 the automatic inverse filtering algorithm will be recalled, and the one dimensional algorithm will be extended to two dimensional signals. In Section 3 the algorithm will be checked on images, and it will be shown that the proposed optimization technique is useful for two dimensional signals as well.

\section{Inverse filtering}

\subsection{Automatic deconvolution}

The optimal reconstruction is defined for which the sum of squared error is minimal. The error function can be written both in time- and frequency domain, utilizing Parseval's theorem:

$$
\begin{aligned}
E E & =\sum_{x=0}^{N_{x}-1} \sum_{y=0}^{N_{y}-1}\left(o(x, y)-o_{e s t}(x, y)\right)^{2} \\
& =\frac{1}{N_{x} N_{y}} \sum_{f_{x}=0}^{N_{x}-1} \sum_{f_{y}=0}^{N_{y}-1}\left|O\left(f_{x}, f_{y}\right)-O_{e s t}\left(f_{x}, f_{y}\right)\right|^{2},
\end{aligned}
$$

where $N_{x}$ and $N_{y}$ are the dimensions of the sampled image. The core of the last sum in (4) can be further written by substituting (3) and (2) into $O_{e s t}\left(f_{x}, f_{y}\right)$ :

$$
\begin{aligned}
&\left|O\left(f_{x}, f_{y}\right)-O_{e s t}\left(f_{x}, f_{y}\right)\right|^{2}= \\
& \mid O\left(f_{x}, f_{y}\right)-O\left(f_{x}, f_{y}\right) P S F\left(f_{x}, f_{y}\right) K\left(f_{x}, f_{y}, p\right) \\
& \quad-\left.N\left(f_{x}, f_{y}\right) K\left(f_{x}, f_{y}, p\right)\right|^{2} \\
&=\quad\left|O\left(f_{x}, f_{y}\right)\left(1-P S F\left(f_{x}, f_{y}\right) K\left(f_{x}, f_{y}, p\right)\right)\right|^{2} \\
&+\left|N\left(f_{x}, f_{y}\right) K\left(f_{x}, f_{y}, p\right)\right|^{2} \\
&-2\left|O\left(f_{x}, f_{y}\right)\left(1-P S F\left(f_{x}, f_{y}\right) K\left(f_{x}, f_{y}, p\right)\right)\right| \\
& \cdot\left|N\left(f_{x}, f_{y}\right) K\left(f_{x}, f_{y}, p\right)\right| \cos \left(\varphi\left(f_{x}, f_{y}\right)\right),
\end{aligned}
$$

where $\varphi\left(f_{x}, f_{y}\right)$ denotes the phase angle of the two absolute valued terms in the last sum and $K\left(f_{x}, f_{y}, p\right)$ denotes the inverse filter having parameter(s) $p$ to control the level of noise reduction. The core of the cost function is split into three terms:

$$
E E=E E_{\text {bias }}+E E_{\text {noise }}+E E_{\text {bias,noise }},
$$

where $E E$ denotes the energy of the error, subscript bias stands for the bias of the estimate, subscript noise for the noise while bias, noise denotes their cross connection. The following approximations will be used to compute the cost function.

- the $E E_{\text {bias,noise }}$ term will be neglected, and

- instead of the absolute values of the signal and noise spectra an approximate spectral model will be substituted into $O\left(f_{x}, f_{y}\right)$ and $N\left(f_{x}, f_{y}\right)$.

The cost function is then the following:

$$
\begin{aligned}
\operatorname{cost} & =\frac{1}{N_{x} N_{y}} \sum_{f_{x}=0}^{N_{x}-1} \sum_{f_{y}=0}^{N_{y}-1}\left|O_{\bmod }\left(f_{x}, f_{y}\right)\right|^{2} \\
& \cdot\left|1-P S F\left(f_{x}, f_{y}\right) K\left(f_{x}, f_{y}, p\right)\right|^{2} \\
& +\frac{1}{N_{x} N_{y}} \sum_{f_{x}=0}^{N_{x}-1} \sum_{f_{y}=0}^{N_{y}-1}\left|N_{\bmod }\left(f_{x}, f_{y}\right)\right|^{2}\left|K\left(f_{x}, f_{y}, p\right)\right|^{2}
\end{aligned}
$$

where subscript mod denotes a frequency domain model of the absolute value of the spectra of corresponding signals. The cost function has to be minimized with respect to parameter(s) $p$ in the inverse filter. The new cost function does not require the knowledge of the intensity of the object, only an approximate model has to be provided for its absolute value of spectrum.

\subsection{Fourier transform of the signals}

The cost function (7) requires the calculation of frequency domain data. Convolution in the frequency domain corresponds to multiplication, however, convolution 


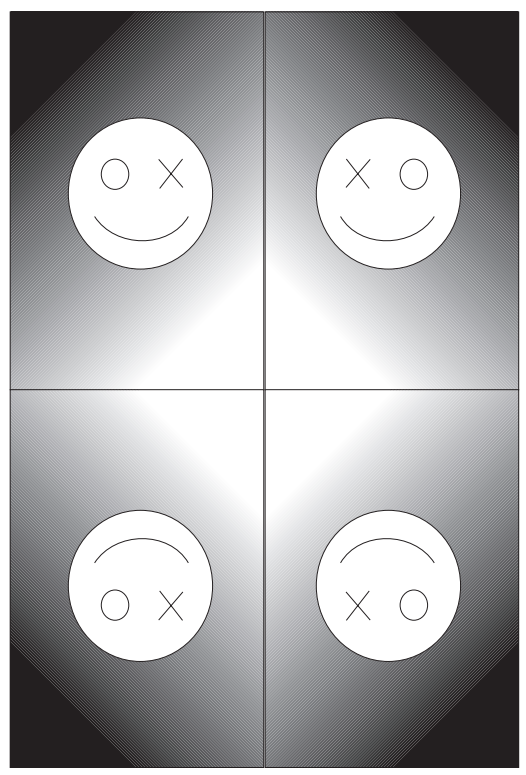

Figure 1. Extension of images to reduce the effect of intensity step on opposite edges

becomes circular. For time domain transient data this effect is usually reduced by padding zeros to the back of the record before computing the Fourier transform. Images, however, are not transient signals; two points on opposite edges have different intensities. To reduce the effect originated from the intensity step along opposite edges we extended the images with their flipped versions (Fig. 1).

\subsection{Modeling of signals}

The signal models are built automatically on the same way as it was proposed for one dimensional transient signals [6]. White noise will be assumed for the noise. Its level can be either a priori measured or extracted from the high frequency part of the spectrum of the noisy image. The absolute value of the spectrum of the undistorted image will be modeled iteratively, starting from a rough model (noisy and distorted measurement), and improving it in several steps by substituting the result of the estimated image into the model.

\section{Simulation example}

The picture of Einstein has been scanned (Fig. 2) in gray scale. The size of the picture 512 by 512 pixels, and the grayscale intensity is quantized in 8 bits. Two dimensional Gaussian distribution with standard deviation of 5 pixels was chosen to model the point spread function of the optical system. Uniformly distributed noise simulates the measurement uncertainty. The width of the noise is 1 LSB of the quantizer ( 1 out of 8 bits). The distorted and noisy image is shown in Fig. 3.

An often used technique of signal reconstruction is the regularization of the transfer function $[4,5]$, which takes the following form for two dimensional signals. We used this algorithm to show the capabilities of the proposed optimization technique:

$$
\begin{aligned}
O_{e s t} & \left(f_{x}, f_{y}, \gamma\right)= \\
& \frac{I\left(f_{x}, f_{y}\right) P S F\left(f_{x}, f_{y}\right)^{*}}{\left|P S F\left(f_{x}, f_{y}\right)\right|^{2}+\gamma\left|D\left(f_{x}, f_{y}\right)\right|^{2}} \\
+ & \frac{N\left(f_{x}, f_{y}\right) P S F\left(f_{x}, f_{y}\right)^{*}}{\left|P S F\left(f_{x}, f_{y}\right)\right|^{2}+\gamma\left|D\left(f_{x}, f_{y}\right)\right|^{2}},
\end{aligned}
$$

where $\gamma$ is the regularization parameter which controls the level of noise suppression.

If the noise is not suppressed enough in the inverse filtering process ( $\gamma$ is small in (8)) the reconstruction will be noisy (Fig. 4). Suppressing the noise too much ( $\gamma$ is large in (8)) results a smooth but distorted image (Fig. 5). The optimal level of noise reduction has been calculated with the proposed algorithm. The obtained reconstruction is shown in Fig. 6.

For simulated signals we can check the performance of the result. We calculated also the best reconstruction which can be achieved at all with the chosen inverse filter. It is calculated by minimizing (4) directly:

$$
\begin{aligned}
& \cos t(\gamma)= \\
& \sum_{f_{x}=0}^{N_{x}-1} \sum_{f_{y}=0}^{N_{y}-1}\left|O\left(f_{x}, f_{y}\right)-I\left(f_{x}, f_{y}\right) K\left(f_{x}, f_{y}, \gamma\right)\right|^{2} .
\end{aligned}
$$

Of course it cannot be calculated in general, only for simulated data, since it requires the knowledge of the original undistorted image. The above expression results the reconstruction shown in Fig. 7. The estimated and the true optimum is very close to each other, which validates the usefulness of the proposed algorithm.

\section{Conclusions}

Inverse filtering of optical images were investigated. A model based optimization technique were shown, which has been adapted from the environment of one dimensional transient signals to two dimensional optical images. The performance of the method was shown on simulated signal. We showed the behavior of the deconvolution for a special inverse filter (regularization), however, the optimization method is not limited to this. It can be used to any optical inverse filter, which has a limited number of parameters to optimize. 


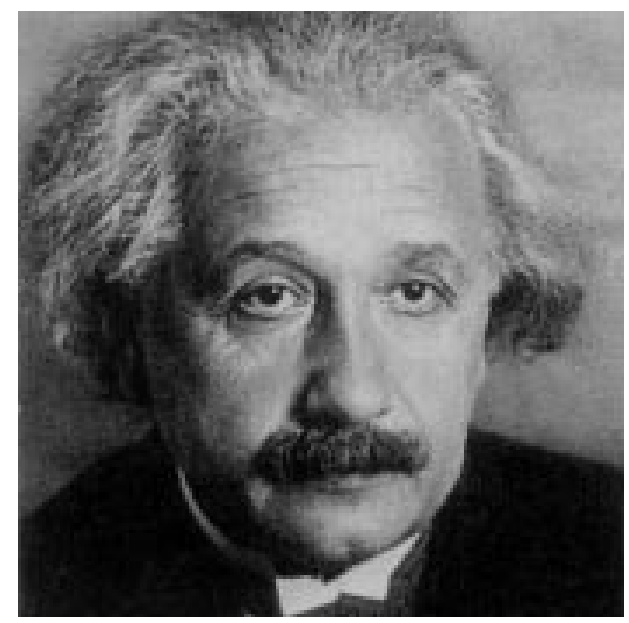

Figure 2. Original picture

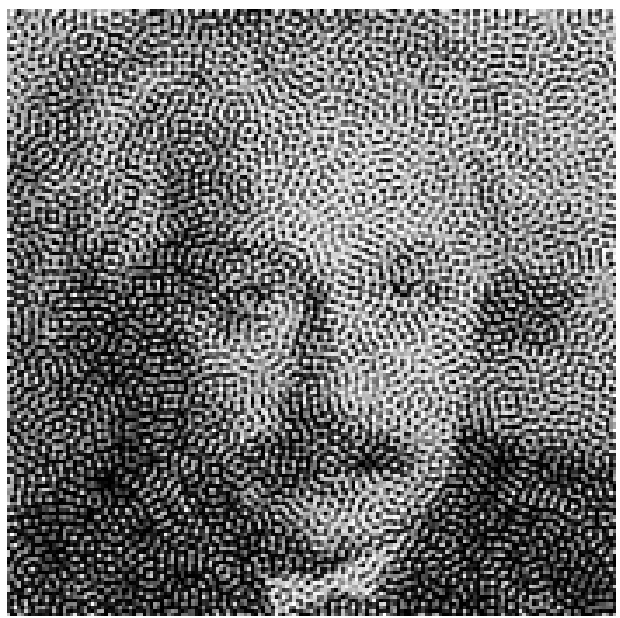

Figure 4. Under regularized reconstruction

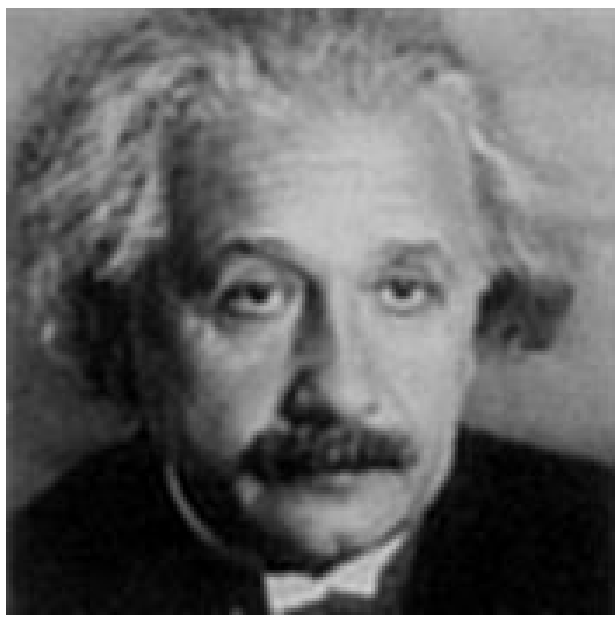

Figure 6. Reconstruction with the proposed algorithm

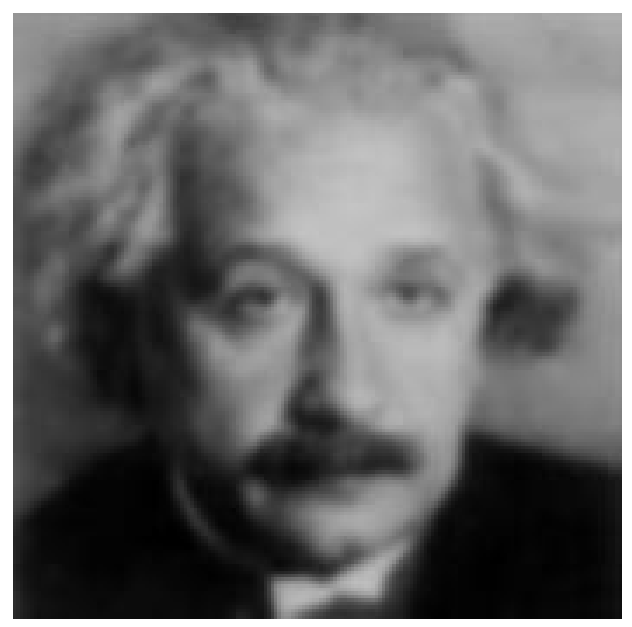

Figure 3. Distorted and noisy image

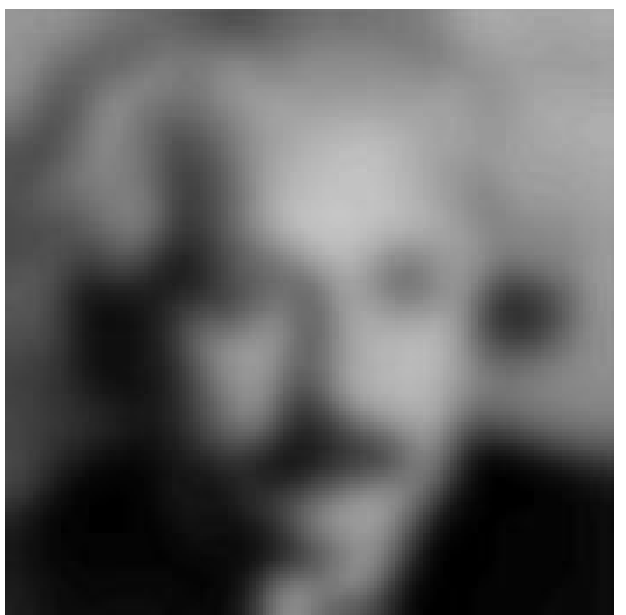

Figure 5. Over regularized reconstruction

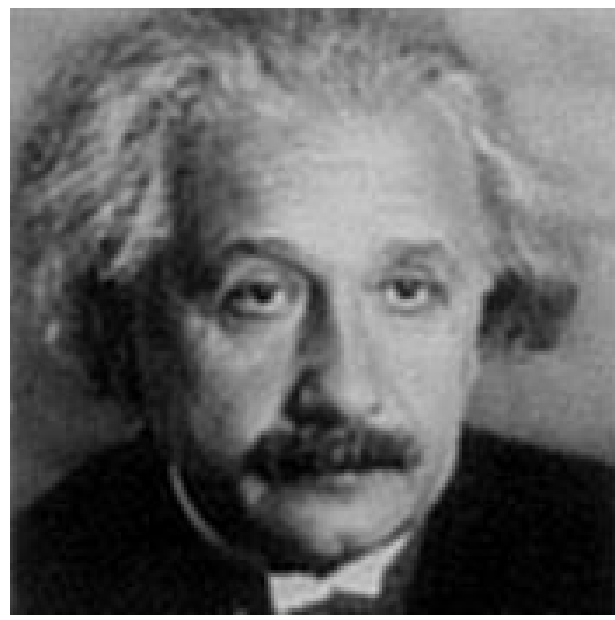

Figure 7. Best reconstruction which can be achieved at all with the given inverse filter 


\section{Acknowledgment}

This research was sponsored by the Hungarian Scientific Research Fund (No. OTKA F026136) and Hungarian Academy of Sciences (Bolyai Janos Scholarship).

\section{References}

[1] D. C. O'Shea, "Elements of modern optical design," John Wiley \& Sons, 1985.

[2] P. M. Duffieux, ”The Fourier transform and its application to optics," John Wiley \& sons, 1983.

[3] S. M. Riad, "The deconvolution problem: an overview," Proceedings of the IEEE, Vol. 74, No. 1, pp. 82-85, 1988.
[4] A. N. Tikhonov and V. Y. Arsenin, "Solution of ill-posed problems," John Wiley \& Sons, Inc., New York, 1977.

[5] N. S. Nahman and M. E. Guillaume, "Deconvolution of Time Domain Waveforms in the Presence of Noise," National Bureau of Standards, Tech. Note 1047, NBS, Boulder, CO. USA, 1981.

[6] Dabóczi T. and I. Kollár, "Multiparameter Optimization of Inverse Filtering Algorithms," IEEE Trans. on Instrumentation and Measurement, Vol. 45. No. 2, pp. 417-421, 1996.

[7] Deyst, J. P., N. G. Paulter, T. Dabóczi, G. N. Stenbacken, T. M. Souders, "A Fast Pulse Oscilloscope Calibration System," IEEE Trans. on Instrumentation and Measurement, Vol. 47, No. 5, pp. 1037-1041, 1998. 